\title{
DISCUSSION
}

\section{Determination of the coefficient of consolidation using a least squares method}

\author{
A. H. C. CHAN (2003). Géotechnique 53, No. 7, 673-678
}

I. G. Doran and J. D. McKinley, Queen's University Belfast The paper proposes the use of an approximation, suggested by Hansen, to the exact analytical solution of the Terzaghi consolidation equation, as the basis for the determination of the coefficient of consolidation from the results of a consolidation test using a least squares error adjustment procedure. This is an area of considerable practical interest. The author seems to be unaware of the paper by Fox (1948), in which it is shown that the solution of the Terzaghi consolidation equation is given to a high degree of accuracy by the approximation

$$
\begin{aligned}
& U=\sqrt{\frac{4 T_{\mathrm{v}}}{\pi}} \quad 0 \leqslant T_{\mathrm{v}} \leqslant 0.2 \\
& U=1-\frac{8}{\pi^{2}} \mathrm{e}^{-\frac{\pi^{2}}{4} T_{\mathrm{v}}} \quad T_{\mathrm{v}}>0.2
\end{aligned}
$$

In fact, equations (1) and (6) of the paper are the same as equations (19a) and (19b) above, respectively, although Fox proposed that the transition point was $T_{\mathrm{V}}=0 \cdot 2$, which corresponds to $U \approx 50 \%$ and not the $U \approx 60 \%$ (i.e. $T_{\mathrm{v}} \approx$ $0 \cdot 286$ ) stated in the paper. Corresponding values of $U$ and $T$ are shown in Table 6, which is an extension of Table 1 in the paper. Clearly, the values calculated from Fox's approximation are sufficiently accurate for any likely application, and are also significantly more accurate than the Hansen approximation used in the paper.

The accuracy of Fox's approximation is further illustrated in
Fig. 4, which shows the difference between the approximate value of $U$ and the exact value, relative to the exact value, as a function of time factor, for several different approximations. This includes the series solution (equations (4) and (5) in the paper) truncated at different values of the index $m$. In this discussion, 'exact' values have been calculated from the series solution summing up to $m=10000$. This is straightforward if the series solution is embedded in a user-defined function in Microsoft Excel using a FOR ... NEXT loop.

The estimation of the consolidation parameters using Fox's approximation can be achieved on a Microsoft Excel spreadsheet using the Solver tool to determine the curve fit that gives the least square error (Doran \& McKinley, in preparation), using a simple $\operatorname{IF}(. .$.$) function. We have also applied$ Solver to the series solution to the Terzaghi consolidation equation, as given by equations (4) and (5) in the paper, and found that in practice about five terms give sufficient accuracy for the interpretation of typical consolidation test results. However, in our experience manipulating even this restricted number of terms is a tedious process unless the series solution is embedded in a user-defined function in Microsoft Excel, whereas the use of Fox's approximation is straightforward and gives satisfactory results.

Figure 4 suggests that improved versions of Fox's approximation can readily be established by the incorporation of extra terms from the series solution. Taking just the first term of the series, $m=0$, a more accurate representation of Fox's approximation is

Table 6. Comparison of various approximate equations with the series solution to the

\begin{tabular}{|c|c|c|c|c|c|}
\hline \multicolumn{2}{|c|}{ Series solution, $m=10000$} & \multicolumn{2}{|c|}{ Hansen solution } & \multicolumn{2}{|c|}{ Fox solution } \\
\hline$T_{\mathrm{v}}$ & $U$ & $U$ & Diff. & $U$ & Diff. \\
\hline $0 \cdot 001$ & $0 \cdot 0357$ & $0 \cdot 0355$ & $-0 \cdot 0002$ & $0 \cdot 0357$ & $0 \cdot 0000$ \\
\hline $0 \cdot 010$ & $0 \cdot 1128$ & $0 \cdot 1122$ & $-0 \cdot 0006$ & $0 \cdot 1128$ & $0 \cdot 0000$ \\
\hline $0 \cdot 020$ & $0 \cdot 1596$ & $0 \cdot 1587$ & $-0 \cdot 0008$ & $0 \cdot 1596$ & $0 \cdot 0000$ \\
\hline $0 \cdot 030$ & $0 \cdot 1954$ & $0 \cdot 1944$ & $-0 \cdot 0010$ & $0 \cdot 1954$ & $0 \cdot 0000$ \\
\hline $0 \cdot 040$ & $0 \cdot 2257$ & $0 \cdot 2245$ & $-0 \cdot 0012$ & $0 \cdot 2257$ & $0 \cdot 0000$ \\
\hline $0 \cdot 050$ & $0 \cdot 2523$ & $0 \cdot 2510$ & $-0 \cdot 0013$ & $0 \cdot 2523$ & $0 \cdot 0000$ \\
\hline $0 \cdot 060$ & $0 \cdot 2764$ & $0 \cdot 2749$ & $-0 \cdot 0015$ & $0 \cdot 2764$ & $0 \cdot 0000$ \\
\hline $0 \cdot 070$ & $0 \cdot 2985$ & $0 \cdot 2969$ & $-0 \cdot 0016$ & $0 \cdot 2985$ & $0 \cdot 0000$ \\
\hline $0 \cdot 080$ & $0 \cdot 3192$ & $0 \cdot 3174$ & $-0 \cdot 0017$ & $0 \cdot 3192$ & $0 \cdot 0000$ \\
\hline $0 \cdot 090$ & $0 \cdot 3385$ & $0 \cdot 3367$ & -0.0019 & $0 \cdot 3385$ & $0 \cdot 0000$ \\
\hline $0 \cdot 100$ & $0 \cdot 3568$ & $0 \cdot 3548$ & $-0 \cdot 0020$ & $0 \cdot 3568$ & $0 \cdot 0000$ \\
\hline $0 \cdot 200$ & $0 \cdot 5041$ & $0 \cdot 5007$ & -0.0034 & $0 \cdot 5046$ & $0 \cdot 0005$ \\
\hline $0 \cdot 300$ & $0 \cdot 6132$ & $0 \cdot 6094$ & $-0 \cdot 0038$ & 0.6134 & $0 \cdot 0001$ \\
\hline $0 \cdot 400$ & 0.6979 & 0.6958 & $-0 \cdot 0021$ & 0.6979 & $0 \cdot 0000$ \\
\hline $0 \cdot 500$ & $0 \cdot 7640$ & $0 \cdot 7647$ & $0 \cdot 0008$ & $0 \cdot 7640$ & $0 \cdot 0000$ \\
\hline $0 \cdot 600$ & $0 \cdot 8156$ & $0 \cdot 8189$ & 0.0034 & $0 \cdot 8156$ & $0 \cdot 0000$ \\
\hline $0 \cdot 700$ & $0 \cdot 8559$ & $0 \cdot 8608$ & $0 \cdot 0049$ & $0 \cdot 8559$ & $0 \cdot 0000$ \\
\hline $0 \cdot 800$ & $0 \cdot 8874$ & $0 \cdot 8927$ & $0 \cdot 0052$ & $0 \cdot 8874$ & $0 \cdot 0000$ \\
\hline $0 \cdot 900$ & $0 \cdot 9120$ & 0.9166 & 0.0046 & $0 \cdot 9120$ & $0 \cdot 0000$ \\
\hline $1 \cdot 000$ & 0.9313 & 0.9347 & 0.0034 & 0.9313 & $0 \cdot 0000$ \\
\hline
\end{tabular}
one-dimensional Terzaghi consolidation equation 


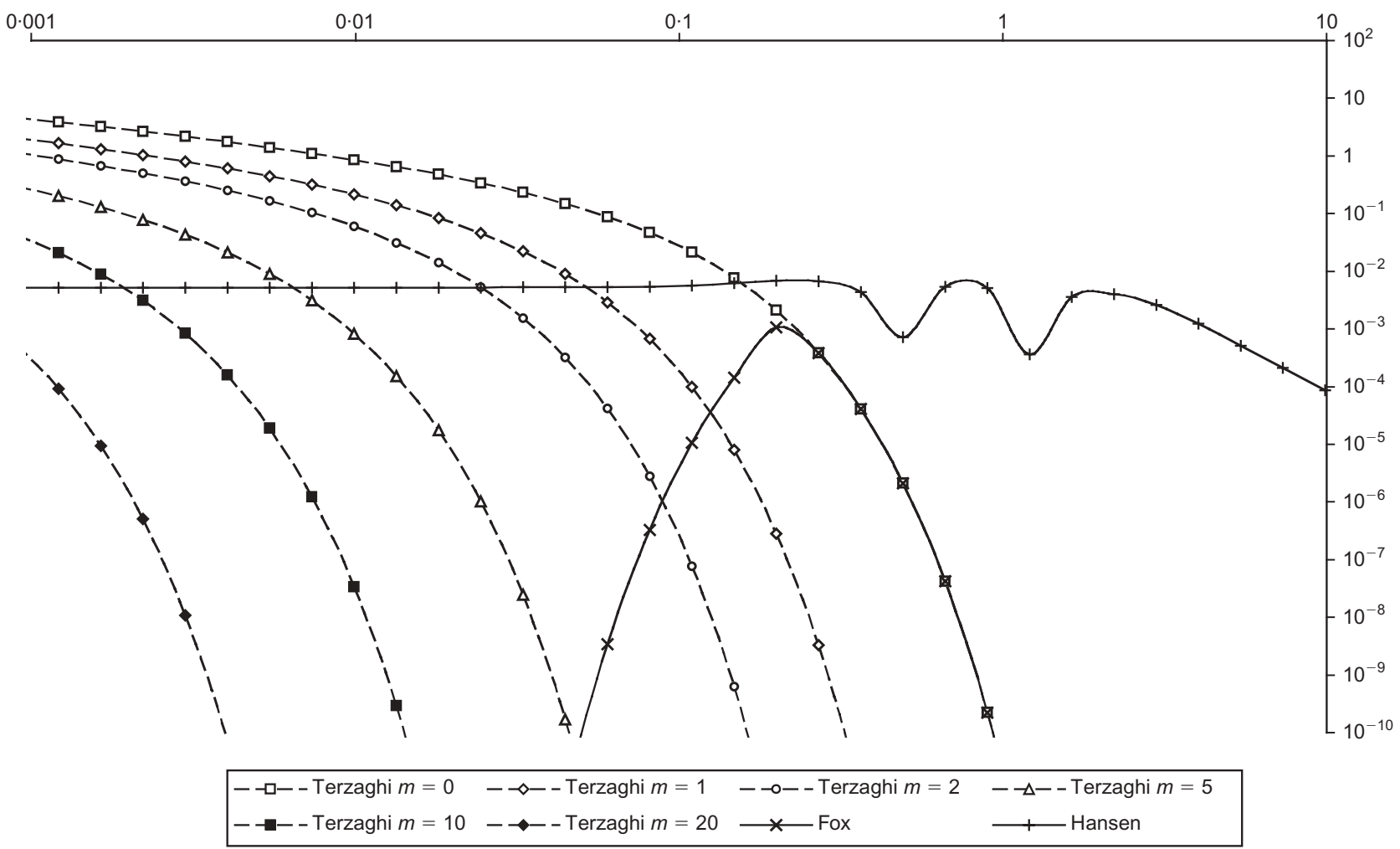

Fig. 4. Comparison of relative difference between various approximate equations with the series solution to the one-dimensional Terzaghi consolidation equation

$$
\begin{aligned}
& U=\sqrt{\frac{4 T_{\mathrm{v}}}{\pi}} \quad 0 \leqslant T_{\mathrm{v}} \leqslant 0 \cdot 213 \\
& U=1-\frac{8}{\pi^{2}} \mathrm{e}^{-\frac{\pi^{2}}{4} T_{\mathrm{v}}} \quad T_{\mathrm{v}}>0.213
\end{aligned}
$$

and at the transition point $U \approx 52 \cdot 1 \%$. For this approximation, the largest relative difference between the approximate solution and the exact solution is $1.5 \times 10^{-3}$. Taking $m=$ 1 , the best approximation is

$$
\begin{aligned}
& U=\sqrt{\frac{4 T_{\mathrm{v}}}{\pi}} \quad 0 \leqslant T_{\mathrm{v}} \leqslant 0 \cdot 125 \\
& U=1-\frac{8}{\pi^{2}}\left(\mathrm{e}^{-\frac{\pi^{2}}{4} T_{\mathrm{v}}}+\frac{1}{9} \mathrm{e}^{-\frac{9 \pi^{2}}{4} T_{\mathrm{v}}}\right) \quad T_{\mathrm{v}}>0.125
\end{aligned}
$$

for which $U \approx 39.9 \%$ at the transition point and the largest relative difference is $3.6 \times 10^{-5}$. Establishing the transition from the parabolic approximation to the truncated series for higher values of $m$ is straightforward, but the approximation in equation (21) is sufficiently accurate for all practical purposes, and that in equation (20) sufficiently accurate for most purposes.

Problems can arise in least squares adjustments when the fitting function is discontinuous. While both approximations (equations (20) and (21)) are continuous in value throughout, to within the numerical accuracy of the calculation, they do have a discontinuity in gradient at the transition point. For $m$ $=0$, equation (20), this discontinuity in gradient is approximately $1.7 \%$ of the gradient at the transition point $T_{\mathrm{v}}=$ $0 \cdot 213$. For $m=1$, equation (21), the discontinuity in gradient is approximately $0.01 \%$ of the gradient at the transition point $T_{\mathrm{v}}=0 \cdot 125$. From our experience of least squares adjustment to fit Fox's approximation with real consolidation data this discontinuity in the gradient in the fitting function does not in practice lead to degenerate adjustments, and either approximation may be used with confidence.

The major difficulty that remains in the solution of the general problem is the determination of the range of readings within a given set of test results that actually comply with Terzaghi consolidation theory, and to which a curvefitting procedure may be applied.

\section{R. A. Day and P. H. Morris, The University of Queensland}

The author has shown that simple linear regression based on equation (8) gives unreliable results. Reasonable results were obtained only when data points at both small and large time values were discarded. An explanation for this is given below.

The author also presents a least squares approach for analysing consolidation test data that involves calculation of complex summations and concludes with the numerical solution of equation (18). The discussion below demonstrates how common numerical tools can be used to simply and directly solve the least squares minimisation problem.

\section{SIMPLE LINEAR REGRESSION}

Consider first the data at large time values - the late data. The author's example displays significant secondary settlement. Equation (8) does not account for secondary consolidation, and thus the late data must be discarded from the regression analysis. Based on conventional methods, the secondary consolidation starts when the reading is about $3.0 \mathrm{~mm}$ (point 13) (Craig, 1995). This is consistent with the findings of the author that better results were obtained if points $15-17$ were excluded.

Consider now the linear regression using the transformed data points $1 / t^{3}$ and $1 / \rho_{t}^{6}$. Fig. 5 shows the result of regression analysis using points $2-10$ plotted on natural 


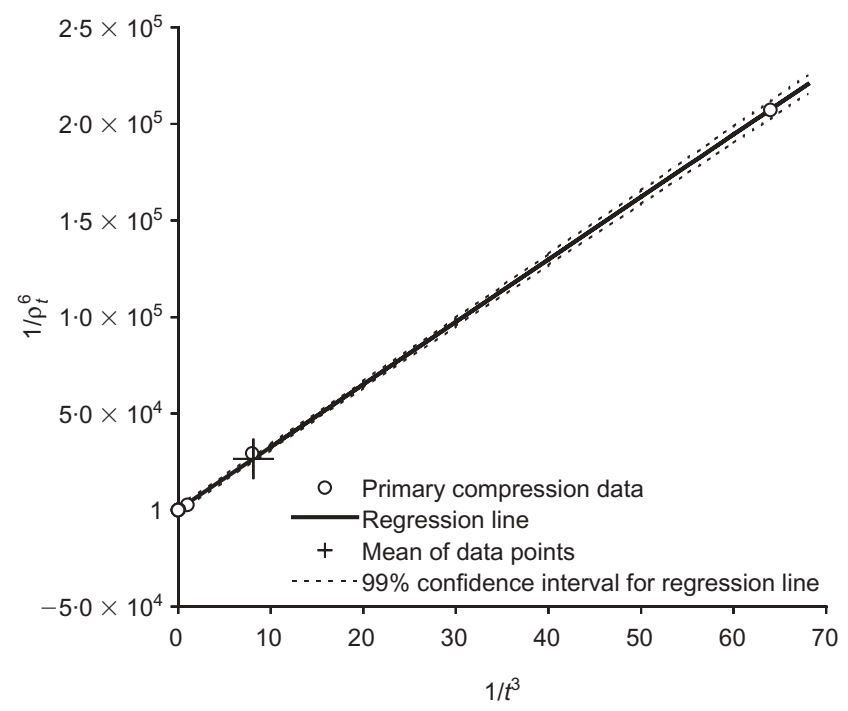

Fig. 5. Result of linear regression using points 2-10 (natural scales)

scales. Points at larger time values were discarded to avoid the issue of secondary consolidation. Note that points 5-10 are so close together they appear as one on this plot. Also shown in Fig. 5 is the $99 \%$ confidence interval for the regression line determined using ordinary statistical methods and assumptions. This means there is a $1 \%$ probability that the true linear relationship does not lie within the bounds shown. The fit is very good: the correlation coefficient is 0.9998. However, in this problem we wish to determine the intercept, or value of the line at $1 / t^{3}=0$. The $99 \%$ confidence interval for the intercept $\left(1 / \rho_{\infty}^{6}\right)$ is from -1330 to 1940. Neglecting negative values, which are physically impossible, this result means there is $99 \%$ confidence that the value of $\rho_{\infty}$ lies between infinity and $0.283 \mathrm{~mm}$. Simple visual inspection of the settlement-time graph gives a much superior result.

Replotting Fig. 5 using logarithmic scales (Fig. 6) enables closer examination of the regression line as $1 / t^{3}$ approaches 0 . It is clear that the regression line neither fits the nearby data points well, nor predicts the intercept accurately.

It is a feature of simple linear regression that the mean values of $1 / t^{3}$ and $1 / \rho_{t}^{6}$ lie exactly on the line. This mean point is also plotted in Figs 5 and 6 . The mean value lies towards the right-hand end of the data set, very close to the

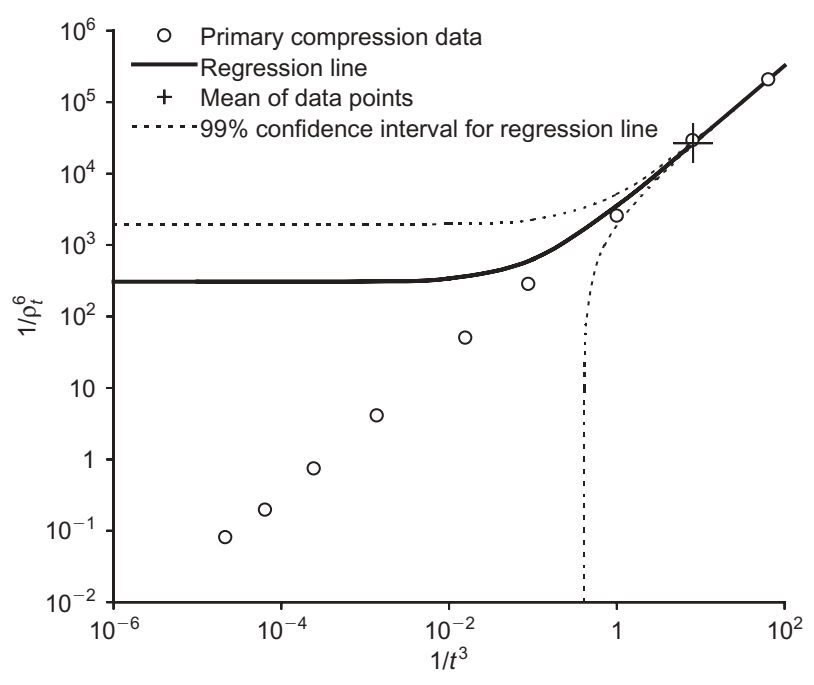

Fig. 6. Result of linear regression using points 2-10 (logarithmic scales) second point plotted (data point No. 3). The first plotted data point is uniquely distant from the mean. Consequently this first point is disproportionately influential in determining the intercept. Discarding early time data points (from the righthand side) does not eliminate this feature of the data; a similar picture emerges in each case as points are successively discarded. The average of the remaining points is always near to the second point from the right because the $1 / t^{3}$ values are distributed approximately uniformly on a logarithmic scale. Fig. 7 shows the different linear regression lines obtained as data points are successively discarded. It can be seen that the predicted intercept switches from negative (impossible) to positive (but too high) values. A reasonably accurate estimate is not obtained until only points $7-10$ are used in the analysis.

As the predicted value of the intercept is used to calculate the value of $c_{\mathrm{v}}$ as well as the final consolidation settlement, an accurate evaluation of the intercept is very important for the consolidation problem. However, it cannot be obtained reliably using the simple linear regression technique described owing to the nature of the consolidation test data, even though it is a good predictor of the trend line. The method is thus intrinsically unsuitable for the analysis of the consolidation test.

\section{LEAST SQUARES FITTING}

The least squares approach presented by the author involves calculation of complex summations, and concludes with the numerical solution of equation (18). A simpler, transparent and elegant approach is to solve the least squares problem directly using numerical techniques commonly available within spreadsheet programs. The problem can be stated as: Minimise the objective function $\Phi$, which is defined as

$$
\Phi=\sum_{i} \varepsilon_{i}^{2}=\sum_{i} w_{i}\left(\rho_{t i}-\rho_{t}\right)^{2}
$$

The weights $w_{i}$ take the value of either 1 or 0 to include or exclude (respectively) the corresponding data point from the objective function. The first data point $(t=0)$ and any data points for which $\rho_{t i}>\rho_{\infty}$ (representing secondary consolidation) must be excluded.

The direct numerical approach does not restrict the theoretical relationship to a simple approximation or single equation. The classical Fourier series solution (equation (4)) is

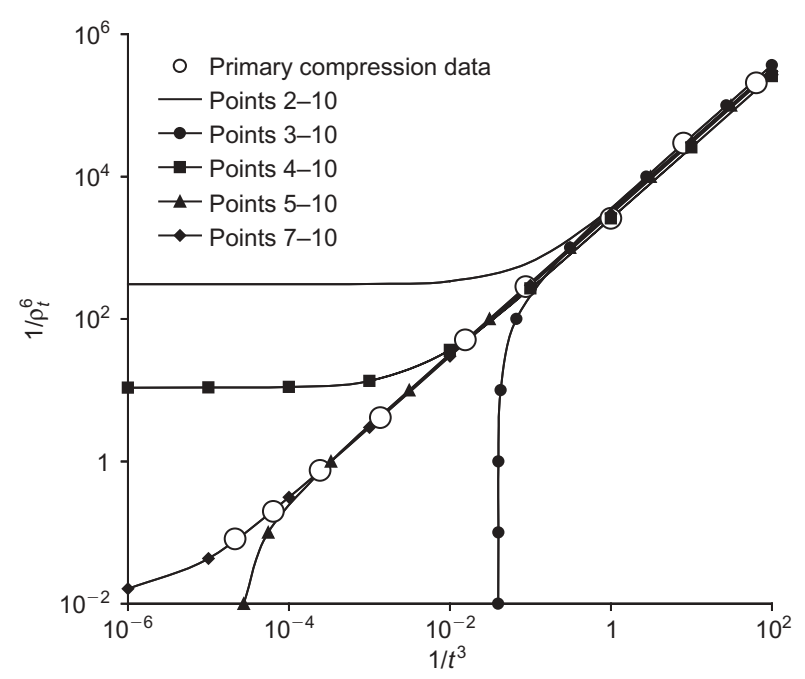

Fig. 7. Results of linear regression using transformed data 
easily used. All three unknown parameters $\left(\rho_{i}, \rho_{\infty}\right.$, and $\left.c_{\mathrm{V}}\right)$ can be determined without more effort or difficulty than finding only two parameters. Fig. 8 demonstrates graphically the good quality of fit that can be obtained using this type of procedure, performed in this case using a common spreadsheet program (Day, 2005), for the analysis of a consolidation test

The least squares approach is simple yet very powerful, and can be easily implemented. It is completely general, and applicable to an enormous range of problems. Multi-variable problems and complex relationships requiring multi-stage calculations can be fitted as easily as simple ones. Discontinuities do not present problems. Multiple relationships can be used to cover the full range of data. For example, equation (23) (Craig, 1995) is very simple to implement for the consolidation test.

$$
\begin{aligned}
& \text { For } U \leqslant 0.6, T_{\mathrm{v}}=\frac{\pi}{4} U^{2} \\
& \text { For } U>0.6, T_{\mathrm{v}}=-0.933 \log (1-U)-0.085
\end{aligned}
$$

\section{Author's reply}

First the author would like to thank the two groups of colleagues who have spent considerable time and effort to investigate and discuss the technical note concerned.

Doran \& McKinley were right in saying that the author is unaware of the paper by Fox (1948), but equations (19a) and (19b) are the same as equations (1) and (6). From Table 1, the $U$ values using equation (1) and equation (6) for $T_{\mathrm{v}}$ values of 0.2 and 0.3 are 0.5046 and $0.6180,0.5051$ and 0.6134 respectively, whereas the exact solutions are 0.5041 and 0.6132 . The author feels that they are close enough for all practical purposes in geotechnical application.

The author agrees that Fox's approximation, whether the transition point is at $U=0.5$ or 0.6 , is sufficiently accurate for any likely application, and is also significantly more accurate than the Hansen approximation used in the technical note. The same conclusion was drawn by the author even before the technical note was published. Therefore the equation pair (1) and (6) with the transition at $U=0.6$ has formed the basis of Tan (2003). Instead of using the 'artificial' example from Craig (1995), 72 sets of real consolidation data have been obtained from three different industrial and academic sources. The results obtained are in good agreement, especially with Taylor's method, for obvious reasons.

The author also agrees that Fox's approximation or the equation pair (1) and (6) with the transition at $U=0.6$ is much more convenient to use than the series solution given by equation (4). Concerning the discontinuity of slope at the interface, the author has the same experience as Doran \&

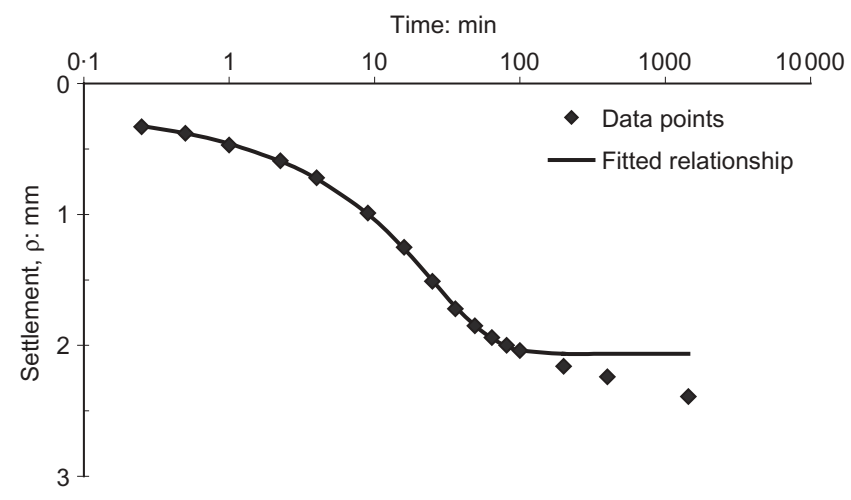

Fig. 8. Comparison of data points and fitted relationship
McKinley. As long as no data point is near the transition, the issue of discontinuity is not of concern. Even if there is a data point at the transition, using one slope or the other does not make any significant difference. For the 72 sets of data analysed, this discontinuity does not seem to cause any major problem.

As for the problems of derivation of parameters from the Terzaghi consolidation, this may arise from the change of stiffness during the consolidation process and significant secondary consolidation settlement. El-Gehani (2004) has tried an automatic procedure that attempted to remove points with significant secondary consolidation. It met with a good degree of success for the data set from Craig (1995), but as the 72 sets of data used do not show significant secondary consolidation behaviour, the result cannot be seen as conclusive. Furthermore, for the 72 data sets tried, the resulting curve fits the data points reasonably well: therefore it can be concluded that the variation of stiffness may not be significant for the 72 sets of data used.

The author would also like to thank Day \& Morris for their detailed study on the simple linear regression scheme that the author tried but failed to investigate further. The author is glad to find that the conclusion from the detailed study is that 'the method is thus intrinsically unsuitable for the analysis of the consolidation test'.

We also tried using a direct minimisation using the same equation as equation (22). It works for some data sets but not all the data sets tried. We found that the direct minimum could be very flat, and Microsoft Excel Solver failed to achieve the absolute minimum. However, we discovered an alternative minimum that is much more suitable for Excel calculation. Instead of solving equation (18), the author realised that it is equivalent to finding the maximum of the function $A\left(c_{\mathrm{v}}\right) / \sqrt{B\left(c_{\mathrm{V}}\right)}$, which is more stable than the solution of equation (18) for Microsoft Excel.

In El-Gehani (2004) we also tried the three-variable method, which uses the initial settlement, the final primary settlement and the coefficient of consolidation as variables. The scheme was devised before the receipt of the discussion from Day \& Morris, and it is good to know that they agree that this is a good way forward. However, from our experience, the initial settlements obtained are very sensitive to the quality of data, and can be very different from the traditional method. The automatic procedure used in ElGehani (2004) was a similar procedure to equation (22).

In conclusion, the author is very grateful for the general interest that the technical note has generated. It is good to know that investigations carried out in three different countries came to similar approaches, with conclusions supporting each other. It is his sincere hope that further development could be made in the automatic determination of the coefficient of consolidation, thus removing operator bias and reducing the time and effort required in oedometer tests, while highlighting soil that has unusual consolidation characteristics.

\section{REFERENCES}

Day, R. A. (2005). http://www.uq.edu.au/robertday/consolidation. Accessed 25 August 2005

El-Gehani, G. A. (2004). New method for the determination of the coefficient of consolidation using a fully automatic least squares procedure. MSc thesis, University of Birmingham.

Fox, E. N. (1948). The mathematical solution for the early stages of consolidation. Proc. 2nd Int. Conf. Soil Mech. Found. Engng, Rotterdam 1, 41-42.

Tan, S. N. (2003). New method for the determination of the coeffcient of consolidation, $c_{\mathrm{v}}$ from the oedometer results. MSc thesis, University of Birmingham. 